Ann. Biol. anim. Bioch. Biophys,, I973, 13 (I), I07-I29.

\title{
ÉTUDE DES INTERRELATIONS ENTRE LES CRITÈRES DE PRODUCTION ET DE COMPOSITION LIPIDIQUE DU LAIT DE CHÈVRE PAR DEUX MÉTHODES D'ANALYSE FACTORIELLE
}

\author{
1). SAUVANT, P.-M. FEHR, F. RODOLPHE*, R. TOMASSONE* et J. DELAGE \\ avec la collaboration technique de P. Guillimin, Annie Rouzeau, \\ Michèle Dorléans et Nicole Sargousse \\ Laboratoire de Zootechnie, I. N. R. A., \\ Institut national agronomique, \\ 16, rue Claude-Bernard, \\ 75005 Paris \\ *Laboratoire de Biométrie, \\ Centre national de Recherches zootechniques, I. N.R. A., \\ 78350 Jouy en Josas
}

\section{RÉSUMÉ}

Les relations entre la production laitière, le taux butyreux, la teneur en matières azotées, les pourcentages des acides gras dans les matières grasses, les quantités secrétées de matières grasses, de matières azotées et d'acides gras, ont été étudiées sur des chèvres ayant mis bas depuis trois mois environ, par deux méthodes d'analyses de données multidimensionnelles : l'analyse en composantes principales et l'analyse factorielle des correspondances. Ces deux méthodes sont décrites afin de dégager leur originalité respective.

A partir des trente critères considérés, les analyses factorielles ont permis de dégager les quatre principaux facteurs de variation suivants : production, longueur de la chaîne carbonée des acides gras, opposition entre les couples d'acides $14: 0,16: 0$ et I8:0, I8: I et opposition entre les caractères azotés et lipidiques du lait.

Par ailleurs, les liaisons entre les caractères de production et de composition, et entre les divers acides gras, la production laitière, le taux butyreux ou la teneur en matières azotées existant dans nos conditions expérimentales, ont pu être "visualisées " de façon très satisfaisante. Les connaissances métaboliques actuelles ont permis d'expliquer certaines de ces relations.

Il a été possible de discriminer trois types de composition lipidique de lait de chèvre dans la population d'animaux étudiés : les laits riches en acides $8: 0$, I0:0, I2:0, les laits riches en acide palmitique et les laits riches en acides en $\mathrm{C}_{18}$. Nous avons constaté que ces méthodes d'analyse factorielle pourraient être utilisées pour sélectionner les chèvres suivant un type donné de composition lipidique du lait, tout en prévoyant les répercussions d'une telle sélection sur les autres caractères.

Bien que l'interprétation détaillée des résultats n'ait pas permis de déceler d'opposition entre les conclusions suggérées par les deux méthodes utilisées, il nous a été possible de discuter les avantages de chacune d'elles et leur aptitude à répondre aux besoins de certaines recherchés métaboliques ou zootechniques. 


\section{INTRODUCTION}

Les recherches sur l'origine des acides gras des triglycérides du lait ont été le plus souvent motivées par la perspective d'augmenter la quantité de matière grasse sécrétée par la mamelle. Mais, dès à présent, les connaissances acquises sur le rôle diététique des différents acides gras, l'attachement grandissant des consommateurs à la notion de qualité et le développement des traitements technologiques des produits laitiers incitent à poursuivre ces recherches afin de maîtriser le spectre des acides gras des matières grasses du lait.

L'origine des acides gras et la synthèse des lipides du lait de ruminant sont actuellement assez bien connues. (FOLLEY et Mac NAUGHT, I96I ; GARTON, I963; LINZELL, I968, JoNES, I969). Les acides gras à I 8 atomes de carbone ont surtout une origine alimentaire mais peuvent provenir, dans certains cas, des réserves adipeuses. A part une fraction de l'acide palmitique pouvant avoir la même origine que les acides en $\mathrm{C}_{18}$ les acides gras à chaîne carbonée plus courte, sont synthétisés par la mamelle à partir des acides acétique et $\beta$-hydroxybutyrique, respectivement produits terminal et dérivé des fermentations du rumen.

Cependant, ces connaissances sur 1'origine des acides gras du lait ne suffisent pas à expliquer tous les phénomènes observés au cours d'expériences nutritionnelles. Parfois, un ou plusieurs acides présentent sans raison apparente une variation différente de celles des acides de même origine ou à nombre de carbone voisin. C'est fréquemment le cas des acides laurique, myristique et palmitique (STULL et al., I966; STORRY et al., I967; GHADAKI, I968 ; FEHR et al., I972). Par ailleurs, le fait d'apporter en plus grande quantité l'un des principaux précurseurs des lipides du lait à la mamelle n'augmente pas obligatoirement la production de la matière grasse. En effet la sécrétion d'un groupe d'acides peut avoir dans certains cas des répercussions sur la quantité produite des autres acides. (MOORE et STEELE, I968; FEHR et al., I972). Enfin l'amplitude et parfois la nature des réponses individuelles aux traitements tendant à modifier la sécrétion lipidique sont fréquemment 1'objet de variations importantes ou imprévisibles, ce qui tend à limiter la portée et rend difficile l'interprétation des études expérimentales entreprises dans ce domaine.

Dan: ces conditions, afin de mieux cerner ces phénomènes qui rendent actuellement malaisée la maîtrise de la production quantitative et qualitative des acides gras du lait, il nous a semblé opportun d'étudier, en évitant tout dispositif expérimental, les relations existant entre les différents acides gras sécrétés, et entre ceux-ci et divers critères de production. Dans ce but, trente caractères différemment corrélés entre eux ont été enregistrés sur une population de chèvres au même stade de lactation et recevant le même régime.

Dans ce genre de problème, fréquent en recherche, l'étude subjective de la structure de la matrice des corrélations entre ces nombreuses variables paraît difficile et l'ajustement à un modèle linéaire hypothétique privilégiant certaines variables semble arbitraire. En revanche, les techniques d'analyses multidimensionnelles en permettant de résumer un ensemble important de données de façon concise avec une perte minimale d'information paraissent particulièrement bien adaptées aux conditions de notre étude. 
Comme ces méthodes ont encore été assez peu utilisées en recherches zootechniques et nutritionnelles (LEFEBVRE et al., I97I ; HENRY et al., I97I), nous nous proposons de donner quelques éléments théoriques succincts et d'essayer de comparer les deux variantes les plus fréquentes actuellement d'analyses multidimensionnelles : l'analyse en composantes principales et l'analyse factorielle des correspondances afin d'estimer leurs originalités respectives dans ce type de recherches.

\section{MATÉRIEL ET MÉTHODES}

\section{A. - Matériel animal}

Cinquante-cinq chèvres (26 Alpines, 28 Saanen, I Poitevine) du troupeau du domaine de Galle (Département de Génétique animale, I. N. R. A.) âgées de 2 à 4 ans ont participé à cette étude. Elles ont mis bas entre le 20 et le 25 décembre 1969 grâce à la méthode du groupement des chaleurs par l'utilisation d'éponges vaginales (CORTEEL, I97I $a$ et $b$ ).

De la mise bas jusqu'au I 5 avril, les chèvres ont reçu le régime hivernal de début de lactation suivant :

- Foin de prairie artificielle (mélange dactyle-luzerne) de moyenne qualité distribué ad libitum. Sa valeur énergétique est estimée à $0,4^{\circ} \mathrm{UF} / \mathrm{kg}$ après les résultats de l'analyse chimique.

- $\mathrm{r} \mathrm{kg}$ d'ensilage vesce-blé de bonne qualité $(0,14 \mathrm{UF} / \mathrm{kg}-25 \mathrm{~g} \mathrm{MAD})$.

- Aliment granulé $(0,74 \mathrm{UF} / \mathrm{kg}$ - $93 \mathrm{~g} \mathrm{MAD})$ ad libitum. Sa consommation moyenne bien que variable suivant les individus peut être estimée à $2 \mathrm{~kg}$ environ par chèvre. Il renferme $50 \mathrm{p}$. Ioo de luzerne déshydratée, $29 \mathrm{p}$. Ioo d'orge, Io p. Ioo d'avoine, $5 \mathrm{p}$. Ioo de maîs, $4 \mathrm{p}$. Ioo de coprah mélassé, et 2 p. roo de complément minéral vitaminique.

- 0,5 à $0,7 \mathrm{~kg}$ d'un aliment concentré du commerce $(0,99 \mathrm{UF} / \mathrm{kg}-175 \mathrm{~g} \mathrm{MAD} / \mathrm{kg})$.

\section{B. - Techniques expérimentales}

Trois prélèvements de lait ont été effectués proportionnellement à la traite du matin et du soir, le II et le 25 mars, et le 8 avril I970, soit respectivement après 2 mois et demi, 3 mois et 3 mois et demi de lactation.

Le jour du prélèvement, la quantité de lait produite est pesée. Le taux butyreux et la teneur de matières azotées du lait sont respectivement mesurés par les méthodes Gerber et au noir Amido. La composition en acides gras des matières grasses du lait est déterminée par chromatographie en phase gazeuse selon des procédés déjà décrits (DeLAGE et FEHR, i967 et FEHR et al., I972). Seuls les principaux acides gras de la séquence de $8:$ o à $\mathbf{8} 8: 3$ ont été pris en considération dans cette expérience. La variable nommée $\mathrm{I} 7$ n'est pas l'acide $\mathrm{I} 7:$ o mais représente l'ensemble $\mathbf{I} 6: \mathrm{I}$, I6 $: 2$, I $7: 0$ iso, $17: 0, I_{7}: \mathbf{r}$.

\section{C. - Méthodes d'analyse statistique des données}

Les résultats sur les variables suivantes : production de lait (PL), de matières grasses (MG) et azotées (MA), taux butyreux (TB), teneur en matières azotées (TA), numéro de lactation (NL), pourcentage $(8$, 10...) et production $(\underline{0}, \underline{18} \ldots)$ des acides gras caprylique, caprique... ont été analysés par les méthodes factorielles en composantes principales normées et des correspondances. Ces techniques ont été mises au point par Hotelling (1933) et BENZECRI (I964) et décrite récemment par Lebart et FÉNELon (I97I) et TOMASSONE (I970).

\section{Principes généraux.}

La plupart des résultats expérimentaux peuvent se mettre sous forme d'un tableau à double entrée $\underset{\sim}{\mathbb{X}}(n, p)$ de $n$ observations et $p$ variables (tabl. I). Dans notre étude une observation correspond à un animal et chaque variable représente l'un des caractères mesurés. La matrice $X$ représente les coordonnées d'un nuage de $n$ points dans un espace à $p$ dimensions (espace $\mathrm{R}^{p}$ ) dans lequel seront définis les axes factoriels. En confondant l'origine de $\mathrm{R}^{p}$ avec le centre de gravité 
du nuage des $n$ observations, les supports des $p$ vecteurs variables deviennent des sous-espaces vectoriels de $\mathrm{R}^{p}$.

Le cosinus de l'angle formé par 2 des $p$ vecteurs variables est égal au coefficient de corrélation entre les 2 variables considérées (LEFORT, I968). Un groupe de caractères très corrélés apparaît donc comme un "faisceau " d'axes variables. Les analyses factorielles consistent à résumer à chaque fois cette redondance d'information en remplaçant chaque «faisceau " d'axes-variables par un seul axe variable moyen interprétable appelé axe factoriel ou composante principale. On voit donc comment ces analyses aboutissent à une réduction du nombre de variables accompagnée d'une perte minimale d'information. La technique mathématique utilisée fait que ces nouvelles variables sont orthogonales c'est-à-dire statistiquement indépendantes. L'essentiel de l'information peut donc être suivie par la projection des variables originelles et des observations sur les sous-espaces vectoriels déterminés par les axes factoriels prépondérants.

\section{TABLEAU I}

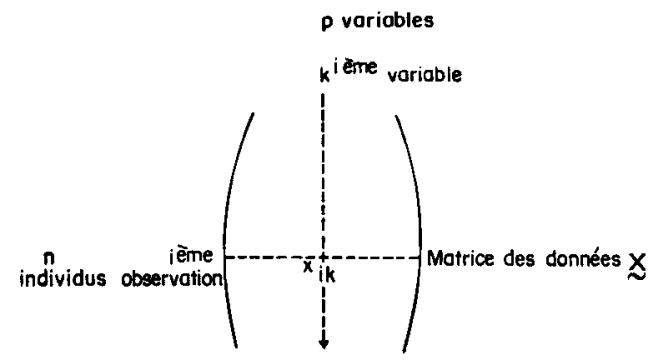

\section{L'analyse en composantes principales normées.}

Cette analyse, utilisée dans un but descriptif, ne suppose pas d'hypothèse sur les lois des variables. Par ailleurs, les résultats restent invariants si une constante quelconque est additionnée ou multiplée à une de ces variables. Afin de comparer des caractères de nature différente le tableau analysé est en fait la matrice $\underset{\sim}{\mathrm{R}}(n x p)$ des variables centrées réduites de terme général :

$$
r_{\imath k}=\frac{x_{i k}-\bar{x}_{, k}}{s_{. k}}
$$

où

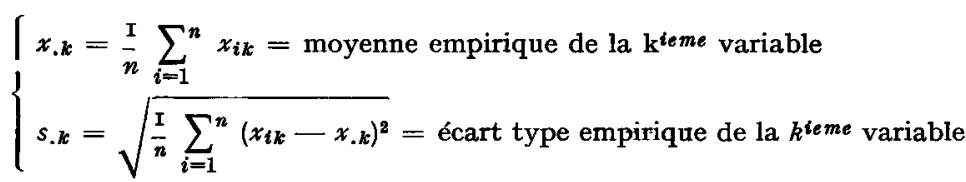

a) Analyse sur les $n$ observations de $R^{p}$

Ces transformations reviennent à munir $\mathrm{R}^{p}$ de la métrique euclidienne identité $(M=I)$,

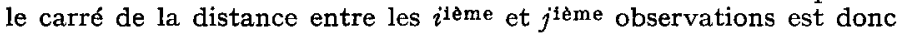

$$
d^{2}{ }_{(i, j)}=\sum_{k=1}^{p}\left(r_{i k}-r_{j k}\right)^{2}
$$

La dispersion ou inertie du nuage par rapport à son centre de gravité, pris ici comme origine est :

$$
\mathrm{I}=\sum_{i=1}^{n} \sum_{k=1}^{p} r_{i k}
$$

La dispersion peut se calculer de façon analogue par rapport à tous les sous-espaces vectoriels de $\mathrm{R}^{p}$. Le problème est de déterminer la droite $\mathrm{D}$ de $\mathrm{R}^{p}$ passant par le centre, suivant laquelle le nouveau nuage est le plus allongé et de ce fait à sa meilleure représentation. Ceci revient à maximiser la somme des carrés des normes des projections des points sur $D$ ou bien ce qui revient au même, l'inertie par rapport à un hyperplan orthogonal à $\mathrm{D}$ à l'origine. Ensuite les droites de 
$\mathrm{R}^{p}$ orthogonales à $\mathrm{D}$ en $\mathrm{O}$ sont recherchées successivement de la même façon dans un ordre décroisant d'inertie expliquée. On détermine ainsi l'ensemble des droites sous-espaces vectoriels de $\mathrm{R}^{p}$ suivant lesquelles le nuage des observations est le plus éclaté.

On démontre que ces droites servent de support aux vecteurs propres rangés dans l'ordre des valeurs propres décroissantes de la matrice carrée symétrique d'ordre $(p x p): \mathbb{R}^{\prime} \underset{\sim}{\mathbb{R}}$. $\left(\mathbb{R}^{\prime}\right.$ est la matrice transposée de $\underset{\sim}{\mathrm{R}}$ ).

Les $p$ vecteurs propres de la matrice $\mathbb{R}^{\prime} \mathbb{R}$ sont orthogonaux et $\frac{\mathrm{I}}{n} \underset{\sim}{\mathbb{R}} \mathbb{R}$ n'est autre que la matrice des corrélations entre les $p$ variables.

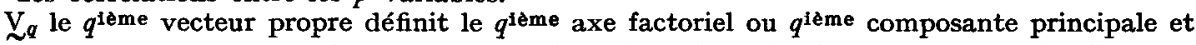
$\lambda_{q}$ la valeur propre correspondante représente l'inertie de la projection du nuage sur le $q^{\text {ieme axe }}$ factoriel. Donc la quantité

$$
\lambda_{q} / \sum_{k=1}^{p} \lambda_{k}
$$

est la part de l'inertie ou variabilité totale du nuage expliquée par la $q^{\text {ième }}$ composante principale.

b) Analyse sur les $p$ variables de $R^{n}$.

On interprète maintenant la matrice des données comme les coordonnées des $p$ points variables dans l'espace $\mathrm{R}^{n}$ repéré par les $n$ axes observation et muni de la même métrique. La démarche théorique est la même que précédemment mais appliquée aux $p$ points variables. Cela revient donc à calculer les vecteurs propres ${\underset{\sim}{\boldsymbol{u}}}_{i}$ rangés par ordre décroissant des valeurs propres $\lambda_{i}$ de la matrice carrée $(n \times n) \underset{\sim}{\mathrm{R}} \mathbb{\sim}^{\prime}$.

On démontre que les premières valeurs propres de $\underset{\sim}{\mathbf{R}}{\underset{\sim}{\mathbf{R}}}^{\prime}$ sont identiques à celles de $\underset{\sim}{\mathbf{R}} \underset{\sim}{\mathrm{R}}$ et que les vecteurs propres correspondants sont liés par la relation :

$$
v_{q}=\frac{\mathrm{I}}{\sqrt{\lambda_{q}}} \mathrm{R}^{\prime} \varkappa_{q}
$$

Dans ces conditions, une variable est bien décrite par un système d'axes factoriels donnés si la norme de sa projection sur le sous-espace vectoriel de $\mathrm{R}^{n}$ engendré par des axes est proche de sa norme dans $R^{n}$, c'est-à-dire si elle peut être considérée comme combinaison linéaire de ces facteurs, le résidu étant dû à des parts de variance expliquées par d'autres facteurs.

Pratiquement les projections des extrémités des vecteurs unitaires des axes variables sur les différentes composantes principales permettent d'évaluer simplement les corrélations variables composantes donc les parts de variance de chaque variable expliquées par les différentes composantes. Les relations possibles entre 2 variables (proximité, orthogonalité, opposition...) sont d'autant plus significatives que leurs projections sur les plans considérés sont éloignées de l'origine.

Les remarques effectuées sur les variables s'appliquent aussi aux individus.

\section{Analyse factorielle des correspondances.}

Cette analyse ne suppose pas d'hypothèse sur les variables qui peuvent par exemple représenter un codage de type [O-I] d'une observation qualitative.

$x$.. étant la somme de tous les termes de matrice $\mathrm{X}$ des données

$$
x_{. .}=\sum_{i=1}^{n} \sum_{k=1}^{p} x_{i k}
$$

$\underset{\sim}{\mathrm{X}}$ est transformé en une matrice $\mathrm{P}$ de terme général

$$
p_{i k}=\frac{x_{i k}}{x . .}
$$

Ce terme général peut s'interpréter comme une fréquence, de même :

$$
\left.\begin{array}{l}
p_{i .}=\sum_{k=1}^{p} p_{i k} \\
p_{. k}=\sum_{i=1}^{n} p_{i k}
\end{array}\right\}
$$

Annales de Biologie animale. - 1973. 
comme des fréquences marginales et

$$
\left.\begin{array}{l}
\frac{p_{i k}}{p_{. k}}=p_{(i / k)} \\
\frac{p_{i k}}{p_{i .}}=p_{(k / i)}
\end{array}\right\}
$$

comme des fréquences conditionnelles.

a) Analyse sur les $n$ observations de $R^{p}$.

Dans $\mathrm{R}^{p}$ on a donc un nuage de $n$ points affectés des masses $p_{i}$, de coordonnées $\left(\frac{p_{i k}}{p_{i}}\right), k=\mathbf{I} \ldots p$ et situés dans un hyperplan à $p$ - I dimensions étant donné que

$$
\sum_{j=1}^{p} \frac{p_{i j}}{p_{i}}=I
$$

La métrique choisie implique que le carré de la distance entre 2 points observations $i$ et $j$ est égal à :

$$
d^{2}{ }_{(i, j)}=\sum_{k=1}^{p} \frac{\mathrm{I}}{p_{. k}}\left(\frac{p_{i k}}{p_{i .}}-\frac{p_{j k}}{p_{. j}}\right)^{2}
$$

Ce qui représente en fait la distance du $\chi^{2}$ entre les deux ensembles de fréquences relatives à $i$ et $j$ (BENzecri, 1964). Cette expression indique qu'il s'agit donc plus d'une analyse des profils que des valeurs, car les pondérations ont supprimé les influences des poids des différentes variables et observations. On démontre que cette distance respecte le principe dit de l'équivalence distributionnelle (LEBART et Fł́nElon, I97I) à savoir que si dans le tableau X, on remplace deux colonnes/deux lignes qui ont même "profil ", par une seule colonne/ligne qui est leur somme terme à terme, l'analyse effectuée est invariante.

Cette expression de la distance montre qu'il est possible d'utiliser une démarche de calculs analogue à celle suivie en composantes principales en prenant pour coordonnées des points $i$ les quantités

$$
\frac{p_{i k}}{p_{i .} \cdot \sqrt{p_{i k}}}
$$

Dans ce cas, le terme général de la matrice $\underset{\sim}{\mathrm{R}}$ devient donc

$$
r_{i k}=\frac{p_{i k}-p_{i . p_{. k}}}{\sqrt{p_{i . p_{. k}}}}
$$

Et les facteurs déterminés sont les vecteurs propres de la matrice $\underset{\sim}{\mathrm{R}} \stackrel{\mathrm{R}}{\sim}$

b) Analyse sur les $p$ variables de $R^{n}$.

On procède de la même manière avec un système de pondération symétrique des points variables, et on calcule les vecteurs propres de la matrice $\mathrm{R} \underset{\sim}{\mathrm{R}}$.

Les valeurs propres ont la même signification qu'en analyse en composantes principales normées. Un des intérêts de cette méthode est que variables et observations sont projetées simultanément sur les mêmes plans factoriels. Sur ces plans 2 points observations/variables proches correspondent à 2 observations/variables ayant des profils comparables sur les variables/observations relativement aux facteurs considérés. Par ailleurs, une observation/variable est d'autant plus proche d'une variable/observation que celle-ci intervient fortement dans le profil de cette observation/variable.

L'originalité des 2 méthodes vient du choix différent de la métrique. De ce fait le traitement plus symétrique des observations et des variables, dans le cas de l'analyse factorielle des correspondances n'existe pas en analyse en composantes principales, ce qui implique 2 techniques đifférentes d'interprétation. 


\section{RÉSULTATS}

\section{A. - Caractéristiques des variables étudiées}

Les moyennes et caractéristiques de dispersion (écart-types, coefficient de variation) des variables étudiées sont rapportées au tableau 2. La production de lait (PL) obtenue à ce stade de la lactation place ce troupeau dans le premier tiers des troupeaux français soumis au contrôle laitier; la teneur en matières azotées (TA) est égale à la moyenne nationale et le taux butyreux (TB) lui est environ inférieur de 7 points (DISSET, r97I). La composition en acides gras du lait est tout à fait comparable à celle précédemment observée chez la Chèvre (DELAGE et FEHR, I967 et FEHR et al., I 972 ). La production laitière et de ce fait les autres facteurs de production qui en dépendent largement ont un coefficient de variation proche de $30 \mathrm{p}$. Ioo, excepté

\section{TABLEAU 2}

Moyennes et caractéristiques de dispersion des variables

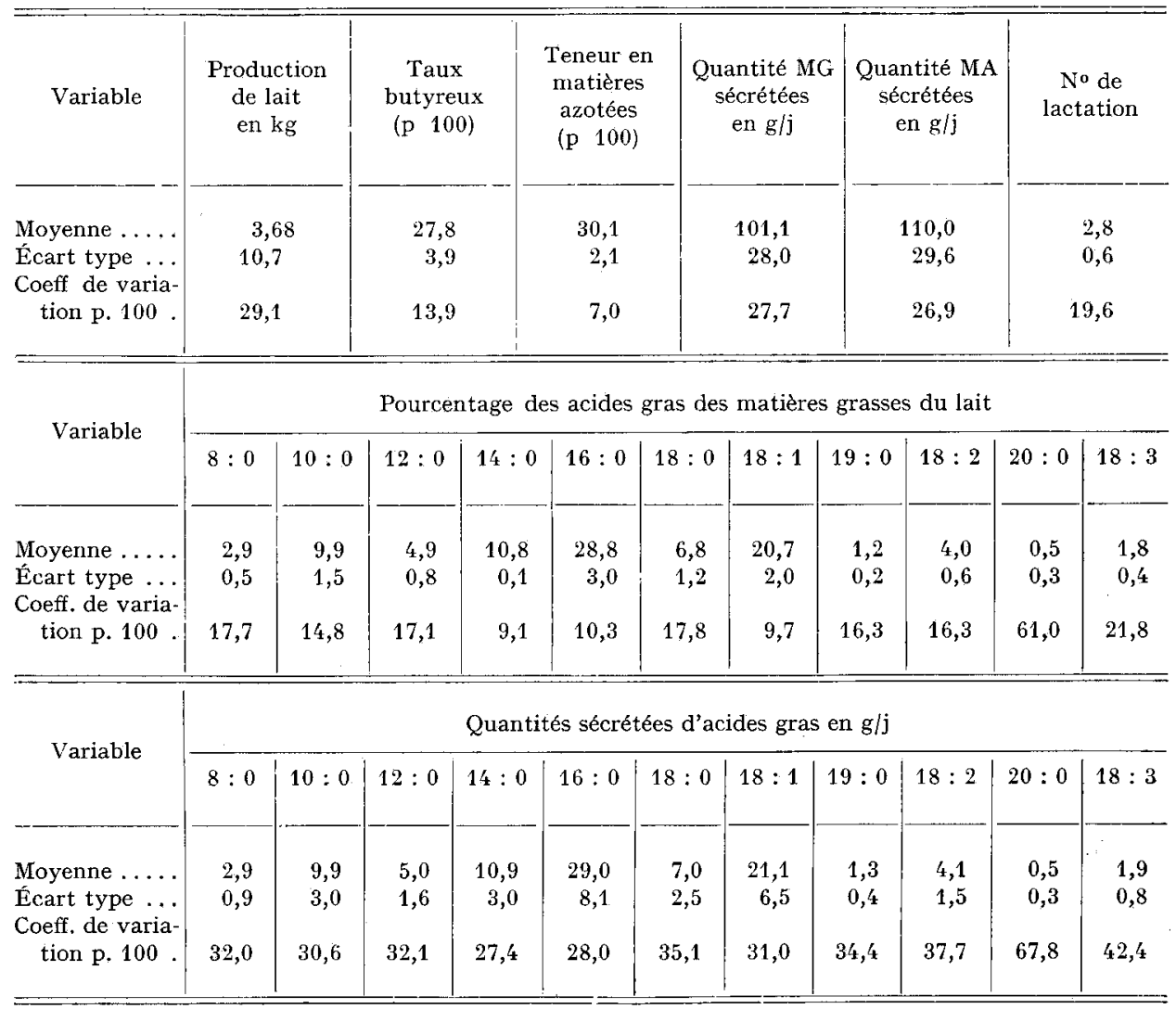




\begin{tabular}{|c|c|c|c|c|c|c|c|c|c|}
\hline & $\begin{array}{l}\mathrm{PL} \\
12\end{array}$ & TB & & & & & & & \\
\hline PL & 1.00 & 14 & TA & & & & & & \\
\hline TB & -.31 & 1.00 & 16 & $\mathrm{MG}$ & & & & & \\
\hline TA & -.39 & .05 & 1.00 & 17 & MA & & & & \\
\hline MG & .86 & .16 & -.37 & 1.00 & $18: 0$ & NL & & & \\
\hline MA & .97 & -.29 & -.21 & .86 & 1.00 & $18: 1$ & 8 & & \\
\hline NL & .35 & -.02 & .04 & .36 & .36 & 1.00 & 19 & 10 & \\
\hline 8 & -.20 & .38 & -.07 & -.00 & -.20 & -.02 & 1.00 & $18: 1$ & 12 \\
\hline 10 & -.20 & .20 & .08 & -.07 & -.17 & -.06 & .69 & 1.00 & 20 \\
\hline 12 & -.20 & .13 & .45 & -.09 & -.10 & .06 & .40 & .74 & 1.00 \\
\hline 14 & -.09 & -.29 & .26 & -.24 & -.07 & .14 & -.08 & .12 & .32 \\
\hline 16 & -.20 & -.01 & -.08 & -.22 & -.26 & .01 & -.32 & -.28 & -.34 \\
\hline 17 & .06 & -.07 & .03 & .02 & .07 & .09 & -.12 & -.34 & -.12 \\
\hline $18: 0$ & .13 & .15 & -.28 & .22 & .09 & -.23 & -.11 & -.28 & -.26 \\
\hline $18: 1$ & .30 & -.11 & -.11 & .21 & .28 & .01 & -.33 & -.54 & -.53 \\
\hline 19 & .21 & -.08 & -.16 & .16 & .18 & -.01 & .08 & -.17 & -.18 \\
\hline $18: 2$ & .57 & -.36 & -.08 & .41 & .61 & .05 & -.22 & -.22 & -.17 \\
\hline 20 & -.05 & .21 & .19 & .02 & -.00 & -.00 & .05 & .11 & .26 \\
\hline $18: 3$ & .39 & -.10 & -.10 & .35 & .42 & .24 & .10 & .01 & .00 \\
\hline 8 & .60 & .32 & -.30 & .83 & .61 & .33 & .53 & .30 & .15 \\
\hline 10 & .67 & .24 & -.26 & .87 & .70 & .33 & .33 & .40 & .29 \\
\hline 12 & .66 & .21 & -.11 & .84 & .71 & .34 & .22 & .32 & .43 \\
\hline 14 & $\begin{array}{r}1.00 \\
.86\end{array}$ & .09 & -.30 & .96 & .87 & .40 & -.01 & -.02 & -.00 \\
\hline & .85 & 1.00 & & & & & & & \\
\hline 16 & .79 & .16 & -.43 & .93 & .76 & .35 & -.11 & -.17 & -.23 \\
\hline & .68 & .90 & 1.00 & & & & & & \\
\hline 77 & .82 & .13 & -.32 & .93 & .82 & .35 & -.02 & -.18 & -.13 \\
\hline & .76 & .90 & .85 & 1.00 & & & & & \\
\hline $18: 0$ & .69 & .19 & -.41 & .84 & .67 & .14 & -.08 & -.23 & 一. 19 \\
\hline & .65 & .75 & .72 & .77 & 1.00 & & & & \\
\hline $18: 1$ & .85 & .11 & -.36 & .95 & .85 & .31 & -.10 & -.23 & -.23 \\
\hline & .71 & .90 & .84 & .91 & .85 & 1.00 & & & \\
\hline 19 & .78 & .09 & -.36 & .88 & .77 & .30 & .03 & -.13 & -.13 \\
\hline & .72 & .81 & .76 & .85 & .81 & .86 & 1.00 & & \\
\hline $18: 2$ & .89 & -.02 & -.31 & .93 & .91 & .29 & -.09 & -.13 & -.11 \\
\hline & .78 & .89 & .81 & .90 & .77 & .93 & .87 & 1.00 & \\
\hline 20 & .26 & .22 & .06 & .37 & .31 & .17 & .01 & .05 & .22 \\
\hline & .46 & .37 & .25 & .33 & .38 & .31 & .32 & .34 & 1.00 \\
\hline $18: 3$ & .78 & .05 & -.27 & .86 & .81 & .40 & .04 & -.03 & -.02 \\
\hline & .77 & .86 & .75 & .85 & .61 & .82 & .79 & .88 & .41 \\
\hline
\end{tabular}

Les corrélations indiquées en caractères italiques sont significatives au seuil $(\mathrm{P}<0,01)$.

Les corrélations indiquées en caractères gras sont significatives au seuil $(P<0,05)$.

Signification des sigles utilisés dans le tableau 3 et les figures $1,2,3,4,5,6$ et 7 .

PL : Production latière, TB : Taux butyreux;

MG : Matières grasses sécrétées ; MA : Matières azotées sécrétées ;

NL : Numéro de lactation. $8,10,12:$ pourcentage en acides $8: 0,10: 0,12: 0 \ldots 8,10,12 \ldots:$ quan tités sécrétées 
$\mathrm{AU} 3$

\section{orrélations}

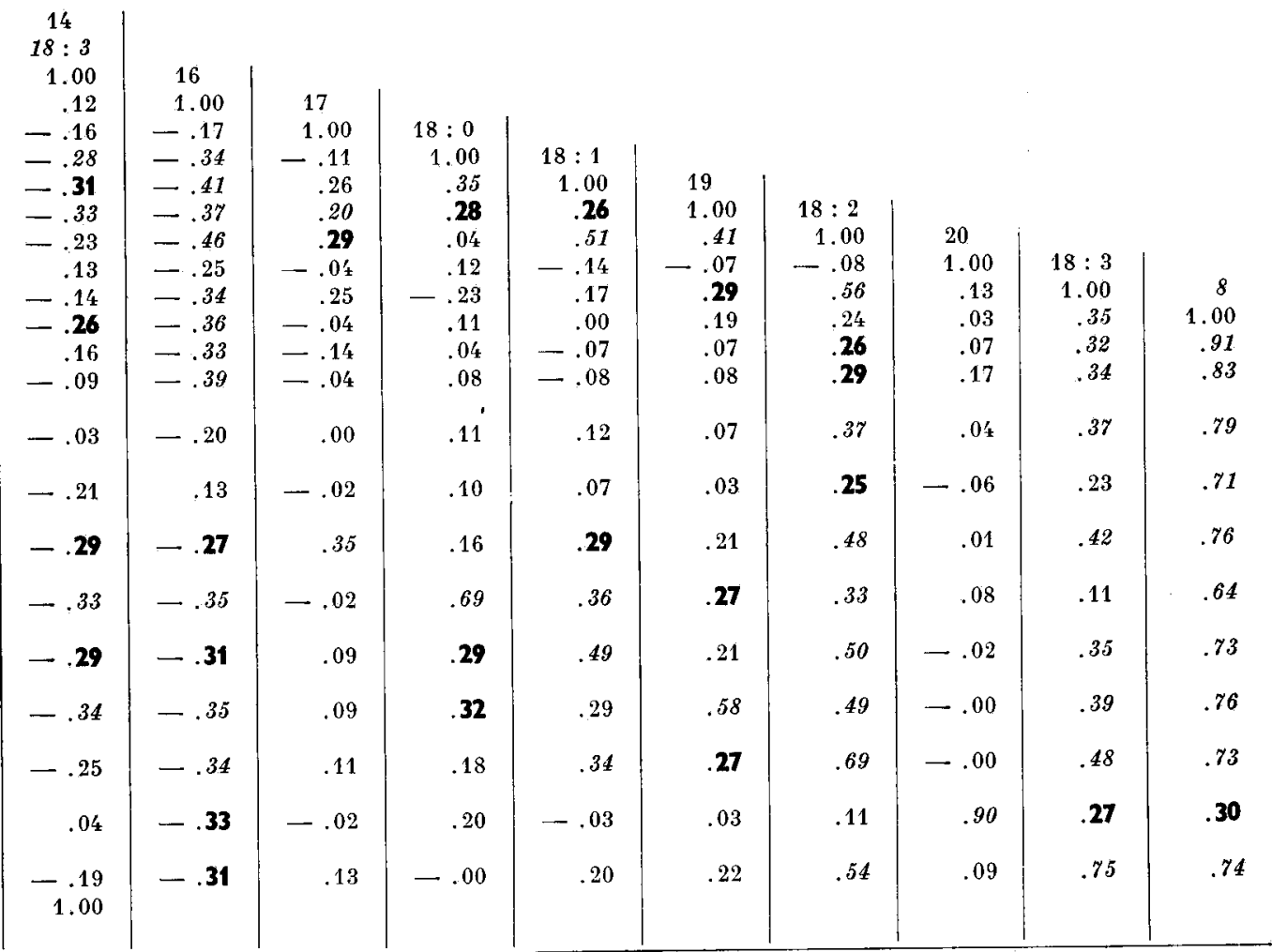

par jour des acides $8: 0,10: 0,12: 0 \ldots$ 
la quantité sécrétée d'acide arachidique $(20: 0)$. La valeur des coefficients de variation des pourcentages d'acides gras dans les matières grasses du lait est sensiblement inférieure : proche de Io $\mathrm{p}$. I00 pour les acides $\mathrm{I} 4: 0, \mathrm{I} 6: 0$, I $8:$ I et comprise entre I4 et $\mathrm{I} 8 \mathrm{p}$. Io0 pour les acides $8: 0$, Io $: 0, \mathrm{I} 2: 0, \mathrm{I} 8: 0, \mathrm{I} 8: 2$.

\section{B. - Analyse en composantes principales}

\section{Matrice des corrélations entre les variables étudiées}

Avant l'extraction des valeurs propres, la matrice des corrélations (tabl. 3) indique déjà que les comportements de certaines variables sont voisins ou opposés. Ainsi les facteurs de production ont des coefficients de corrélation positifs et relativement élevés entre eux. En revanche les corrélations entre les pourcentages des acides à longue chaîne et à courte chaîne ou bien entre la production laitière et les teneurs en matières grasses et en matières azotées sont négatives. La suite de l'analyse permet d'étudier plus finement la structure de cette matrice.

\section{Composantes principales.}

La diagonalisation de la matrice des corrélations a permis d'extraire 30 composantes principales qui expliquent chacune une part indédependante de l'inertie du nuage. Le fait que les cinq premières composantes retenues expliquent presque 80 p. Ioo de l'inertie totale (tabl. 4), rend possible une "visualisation "d'une part encore plus importante de l'information recherchée. Le premier axe factoriel a une position prédominante puisqu'il représente près de $42 \mathrm{p}$. roo de la dispersion totale alors que les quatre axes suivants n'en explique qu'environ 36 p. Iоo.

TABLEAU 4

Explication de la dispersion totale par les cinq axes factoriels en analyse en composantes principales

\begin{tabular}{c|c|c}
\hline \hline Axe factoriel & Dispersion expliquée & $\begin{array}{c}\text { Dispersion expliquée } \\
\text { cumulée }\end{array}$ \\
\hline & & \\
\hline 1 & 42,65 & 42,65 \\
2 & 13,58 & 56,23 \\
3 & 8,08 & 64,32 \\
4 & 7,88 & 72,20 \\
5 & 6,53 & 78,72 \\
\hline
\end{tabular}

3. Représentation des variables par rapport aux composantes principales.

Dans le tableau 5, sont indiquées d'une part les corrélations entre les variables et les 5 composantes principales et d'autre part la proportion d'inertie de chaque variable qui est expliquée par ces cinq composantes. Les caractères de production sont très bien représentés; en effet, au moins $90 \mathrm{p}$. Ioo environ de leur dispersion 
TABLEAU 5

Corrélations entre les variables et les cinq premières composantes principales

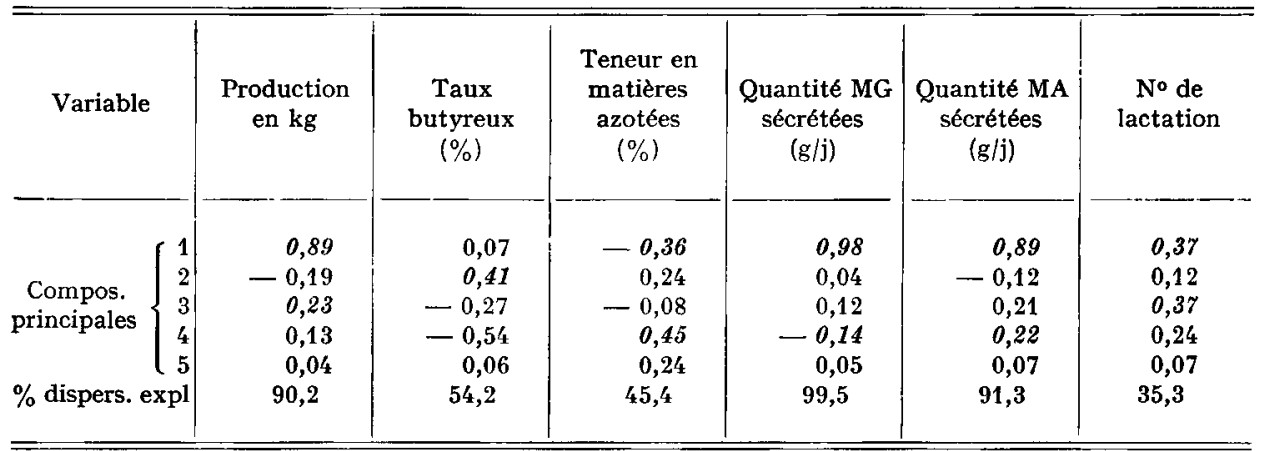

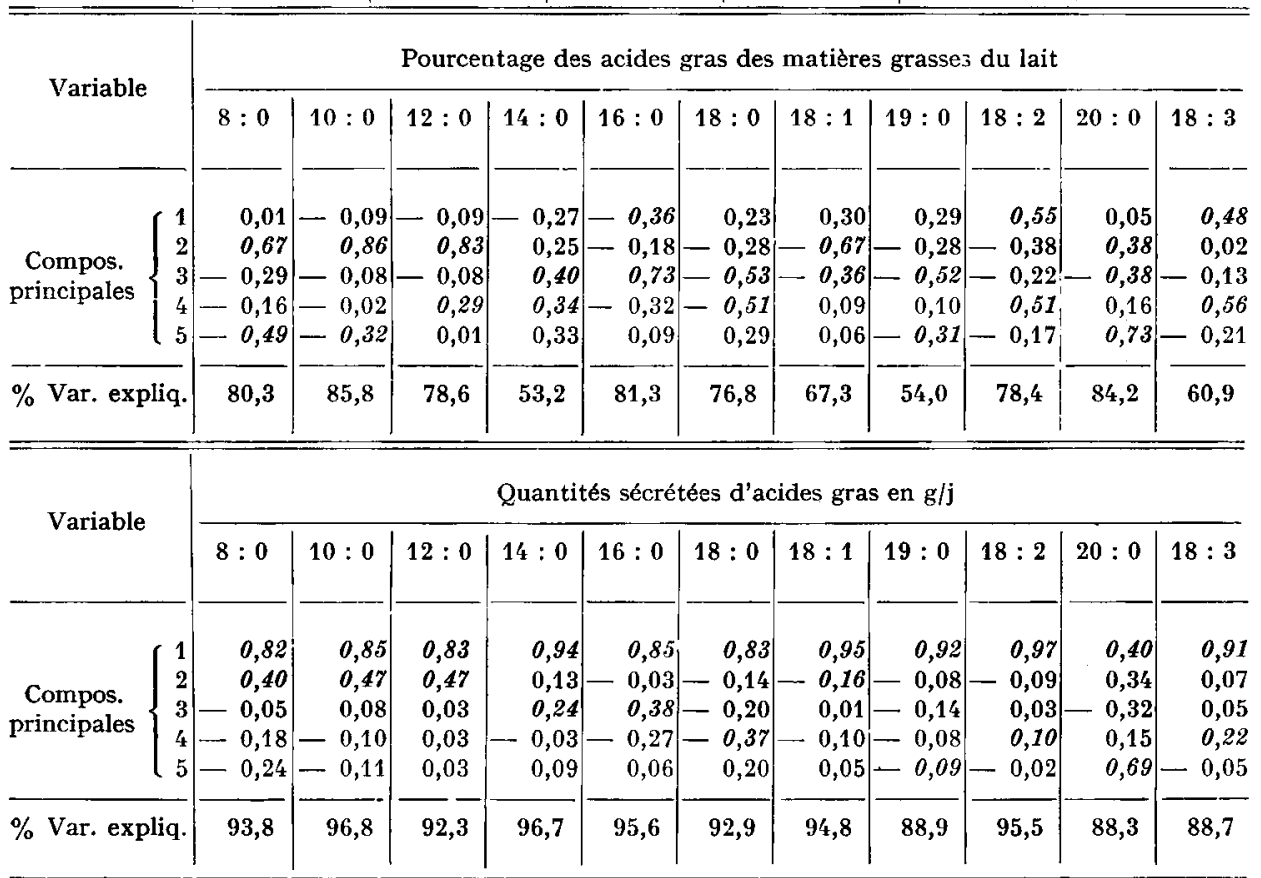

Les corrélations sont indiquées en caractère italique lorsqu'elles correspondent aux deux composantes principales qui expliquent le mieux l'inertie d'une variable.

se trouve expliquée. En revanche, les teneurs en matières grasses et en matières azotées sont médiocrement représentées avec approximativement la moitié seulement de leur inertie expliquée par les cinq facteurs. La qualité de la représentation des pourcentages des acides gras est très variable et paraît indépendante de leur degré de saturation, de la valeur de leur pourcentage et de la longueur de leur chaîne carbonée 
bien que les acides gras courts excepté i'acide myristique ont une légère tendance à être mieux représentés.

Les graphiques $I, 2,3$ représentent les projections des vecteurs unitaires des axes " variables " sur les plans principaux I-2, 2-3, 2-4; ce qui permet de visualiser commodément les relations entre les variables et la qualité de la représenıation de chacune d'elles dans le plan considéré.

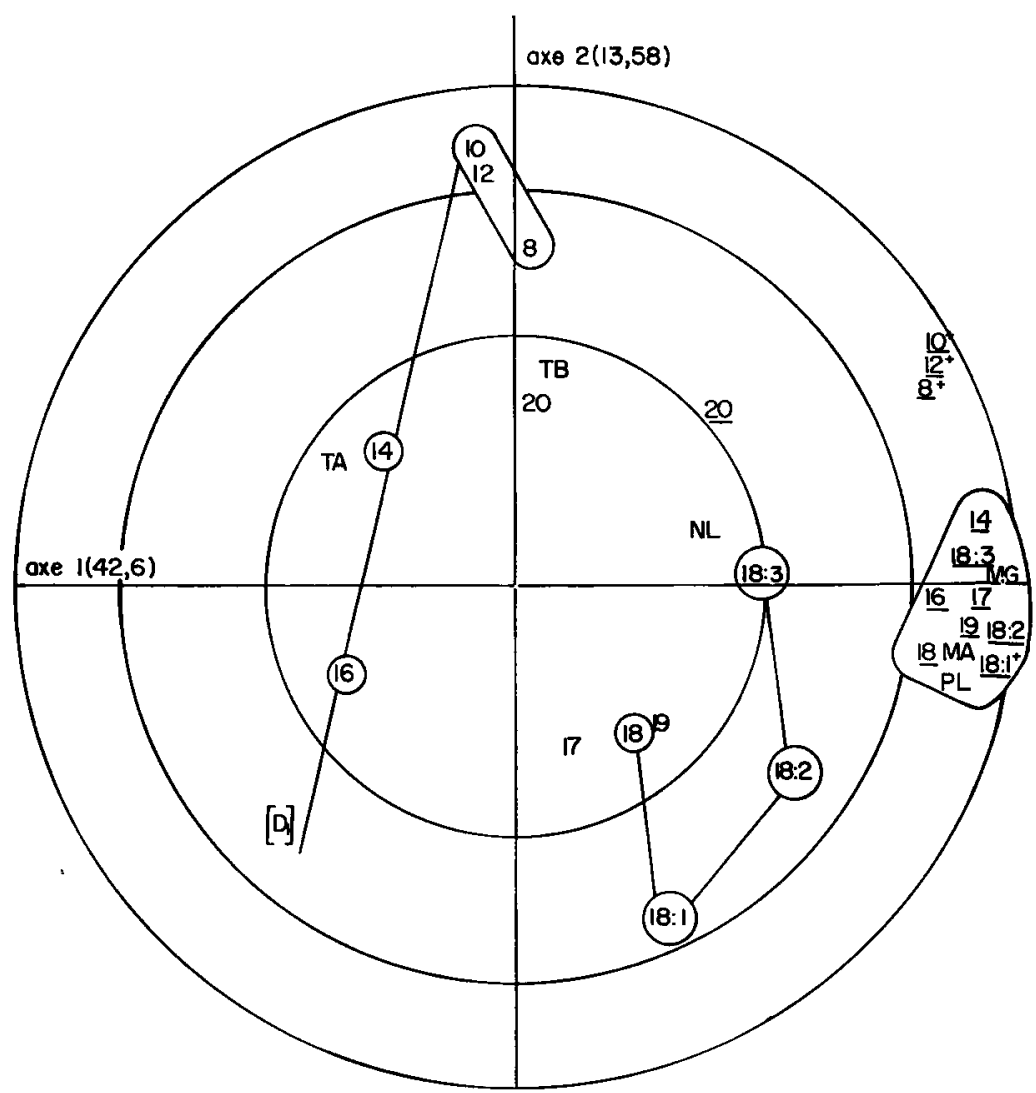

FIG. I. - Plan principal (x-2) de l'analyse en composantes principales

Signification des sigles : cf. tabl. 3

* Les cercles de rayon 0,5 et 0,8 permettent de discriminer à l'œil des variables qui ont moins de 25 p. I0o, de 25 à $64 \mathrm{p}$. 1oo et plus de $64 \mathrm{p}$. 1oo de leur inertie expliquée dans le plan.

Les variables qui sont à leur optimum de représentation dans un plan observé sont signalées par un astérisque.

Dans le graphique I, l'axe factoriel I peut s'identifier au facteur de production. En effet, 1'ensemble des caractères de production se placent autour de la partie positive de cet axe au-delà du cercle 0,8 (64 p. Ioo d'inertie expliquée), alors que les caractères de composition se dispersent dans le reste du plan. Les pourcentages des acides à $\mathrm{r} 8$ atomes de carbone et notamment ceux des acides linoléique et linolénique sont plus proches du caractère production. Les acides plus courts semblent d'autant plus opposés à ce caractère que leur chaîne est longue comme l'indique la droite $D_{1}$. Èn 
outre, le taux butyreux paraît dans ce plan indépendant du caractère production alors que la teneur en matières azotées a tendance à s'y opposer.

L'axe 2 semble classer les acides gras saturés suivant la longueur de leur chaîne à l'exception de l'acide arachidique. Les pourcentages des acides gras courts sont placés à I'opposé de ceux des acides en $\mathrm{C}_{16}$ et $\mathrm{C}_{18}$ alors que l'acide en $\mathrm{C}_{14}$ se situe dans une position intermédiaire à proximité de la teneur en matières azotées et du taux butyreux.

L'axe 3 semble mettre en valeur l'opposition des acides à longue chaîne d'orgine différente : l'acide palmitique et les acides à 18 carbones et dans une moindre mesure, il discrimine les acides en $\mathrm{C}_{18}$ suivant leur degré de saturation (graph. 2). L'axe 4 oppose en particulier les caractères azotés et lipidiques des laits (graph. 3).

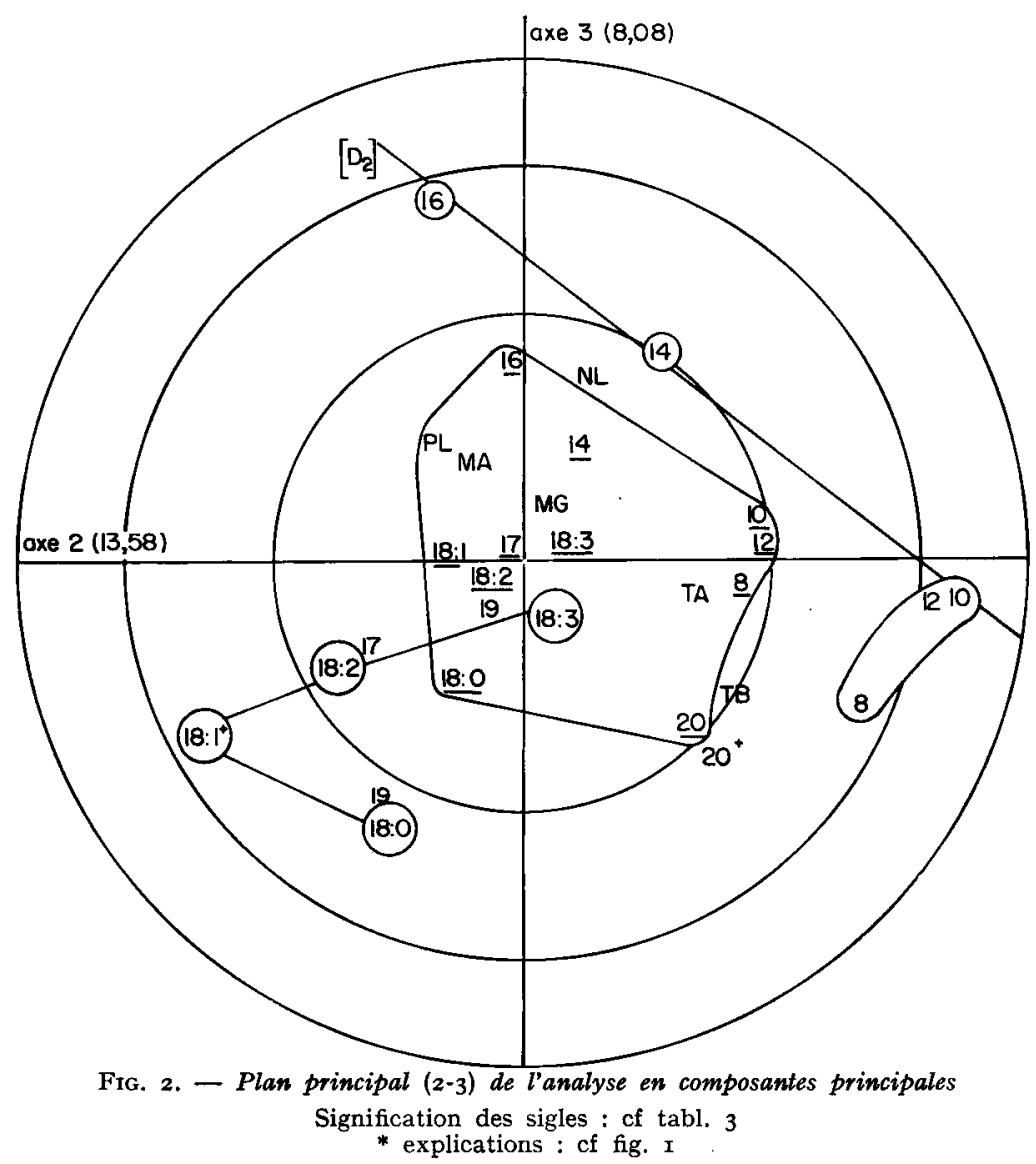

Ainsi, compte tenu de l'importance du facteur production représenté par l'axe $\mathrm{r}$, il est intéressant de se placer orthogonalement par rapport à cet axe, donc indépendamment de ce facteur en considérant la répartition des points variables dans l'espace des composantes $2,3,4$.

Dans le plan 2-3 (graph. 2) les critères de composition sont mieux représentés 
que les critères de production qui restent dans une zone peu significative. Ce plan paraît discriminer trois types principaux de distorsion bien expliqués du spectre des acides gras du lait dans la population étudiée :

- les laits riches en acides gras courts $8: 0$, ro: 0, I2 : 0

- les laits riches en acide palmitique

- les laits riches en acides stéarique, oléique et linoléique.

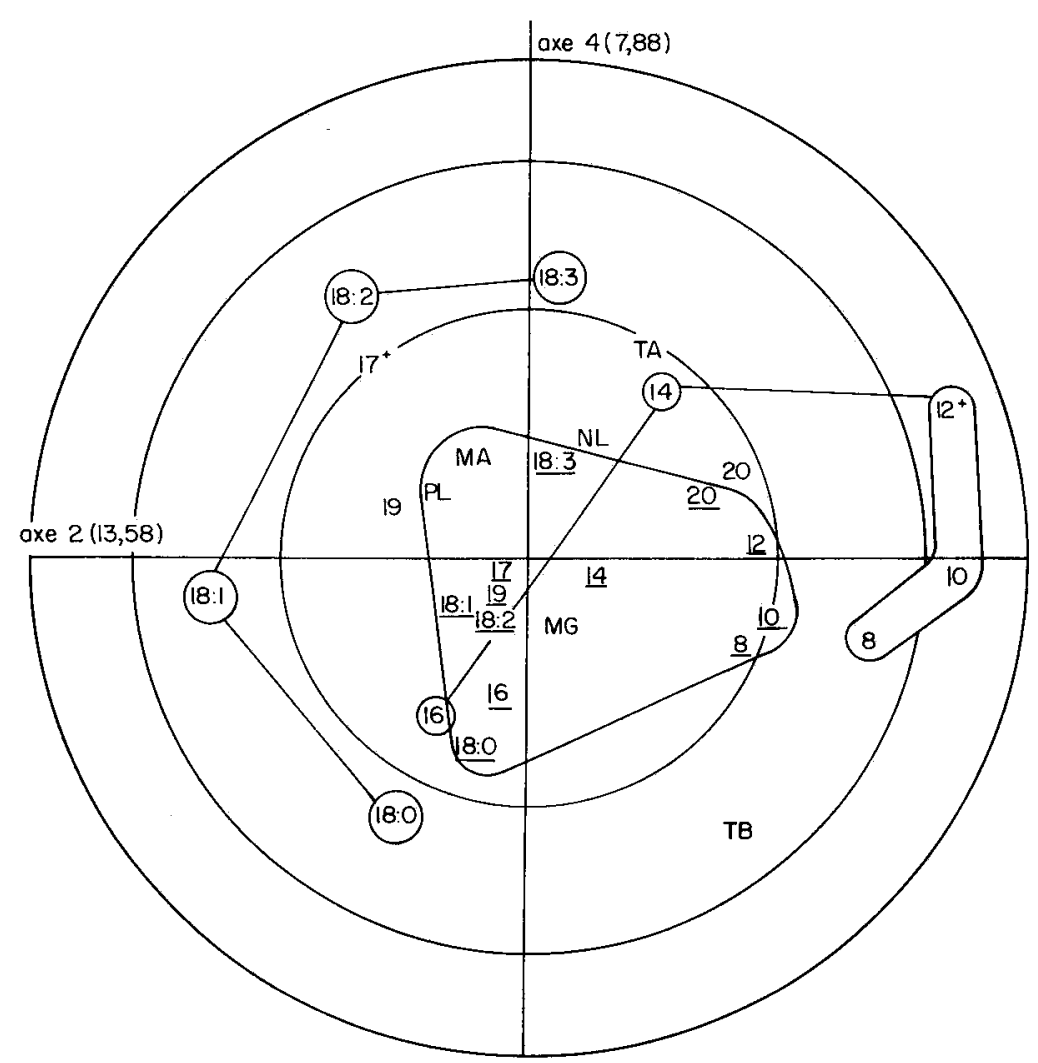

FIG. 3. - Plan principal (2-4) de l'analyse en composantes principales Signification des sigles : cf. tabl. 3

* explications : cf fig. I

Les autres acides se placent dans des positions intermédiaires : I4: o entre les acides courts et l'acide palmitique (droite $\mathrm{D}_{2}$ ) 20 : o et $\mathrm{I} 8: 3$ entre les acides courts et les acides en $\mathrm{C}_{18}$. Le nuage des observations indique que les compositions des laits se répartissent de manière homogène entre ces trois types extrêmes et leurs intermédiaires.

L'adjonction d'une dimension supplémentaire (axe 4) au plan 2-3 (graph. 3) donne la possibilité de préciser comment 1'opposition entre les caractères azotés et lipidiques du lait module les observations précédentes.

La quatrième composante permet d'éclater les groupes d'acides qui jusqu'à maintenant avaient un comportement proche; dans les acides en $\mathrm{C}_{18}$, l'acide stéa- 
rique se retrouve du côté lipidique et les acides linoléique et linolénique du côté azoté ; dans les acides courts, l'acide caprylique se place plutôt du côté lipidique et l'acide laurique du côté azoté enfin l'acide myristique est très proche de la teneur en matières azotées et l'acide palmitique a sur cette composante un comportement très comparable à $\mathrm{C}_{18}:{ }_{0}$ et $\mathrm{C}_{18}:{ }_{1}$.

\section{C. - Analyse des correspondances}

Les 5 axes factoriels représentent plus de $9 \mathrm{I}$ p. Ioo de la dispersion totale du nuage (tabl. 6).

Le premier axe qui représente près de $60 \mathrm{p}$. roo de l'inertie discrimine plus nettement, qu'en composantes principales, les caractères de production et de composition (graph. 4). Comme dans l'analyse précédente, l'axe I sépare les acides à $\mathrm{I} 8$ atomes de carbone et les acides plus courts et de même les acides en $C_{13}$ sont plus proches de la production laitière que les acides à courte chaîne.

TABLEAU 6

Explication de la dispersion totale par les cinq axes factoriels en analyse des correspondances

\begin{tabular}{c|c|c}
\hline \hline Axe factoriel & Dispersion expliquée & $\begin{array}{c}\text { Dispersion expliquée } \\
\text { cumulée }\end{array}$ \\
\hline 1 & $\mathbf{5 9 , 3 0}$ & \\
2 & $\mathbf{1 6 , 2 9}$ & $\mathbf{5 9 , 3 0}$ \\
$\mathbf{3}$ & $\mathbf{7 , 0 2}$ & $\mathbf{7 5 , 5 8}$ \\
4 & $\mathbf{5 , 9 2}$ & $\mathbf{8 2 , 6 1}$ \\
$\mathbf{5}$ & $\mathbf{2 , 7 5}$ & $\mathbf{9 1 , 2 8}$ \\
\hline
\end{tabular}

Si on fait abstraction de ce facteur de production en considérant l'espace des axes $2-3-4$, on constate que la répartition des points variables est assez comparable à celle observée en composantes principales, à condition de faire une rotation sur l'ordre de sortie des axes factoriels. Cependant l'axe 2 oppose plus nettement les caractères lipidique et azoté que l'axe 4 des composantes principales et les productions de lait et de matières azotées y apparaissent en plus étroite relation. Le plan 3-4 (graph. 5) est comparable au plan 2-3 (graph. 2) des composantes principales, toutefois, l'axe 3 des correspondances différencie les acides suivant le nombre de carbones légèrement moins bien que l'axe 2 des composantes principales, surtout pour les acides longs et très courts.

Les variables et les observations ont été projetées sur un même plan dans le graphique 4 afin de mettre en évidence l'un des principaux intérêts de l'analyse des correspondances.

Globalement le nuage des observations apparaît homogène mais les différences individuelles sont nettes : certaines chèvres sont proches des caractères de produc- 


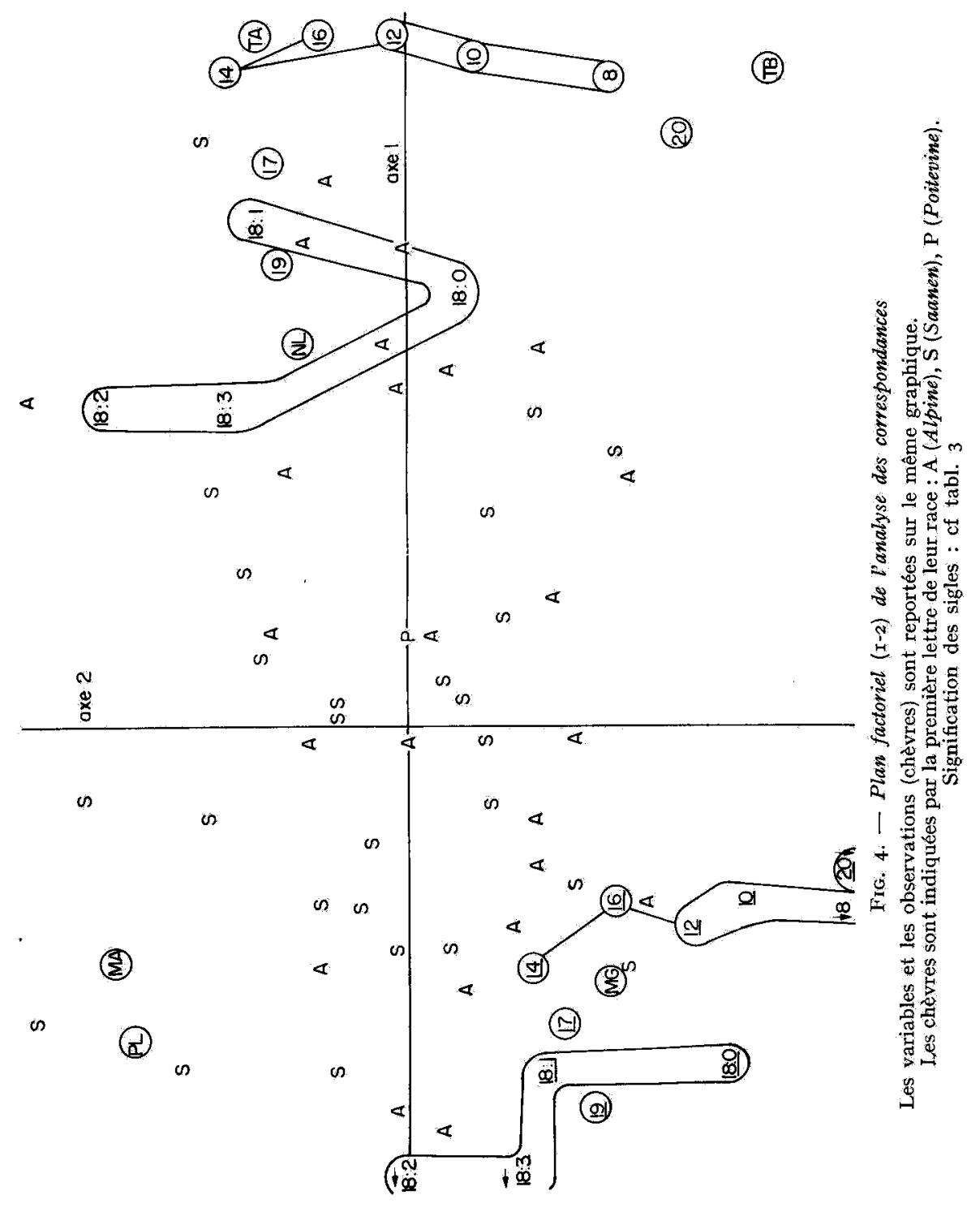


tion, alors que d'autres se placent à proximité des caractères de concentration. Le codage a posteriori des animaux Saanen (S) ou Alpin (A) ne révèle pas de différences évidentes entre ces deux types de chèvres.

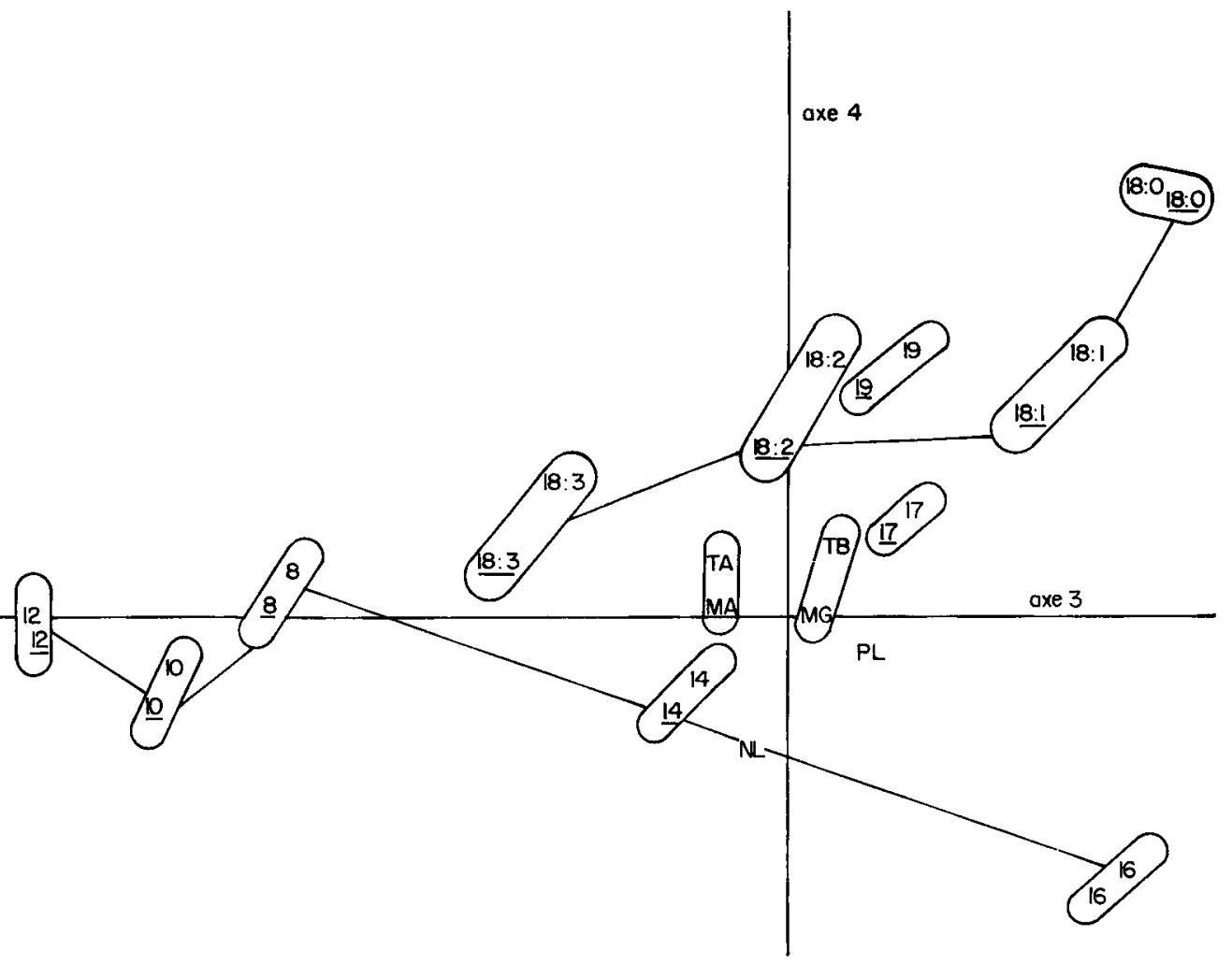

FIG. 5. - Plan factoriel (3-4) de l'analyse des correspondances Signification des sigles : cf. tabl. 3

\section{DISCUSSION}

L'objectif de ce travail a été limité volontairement à l'étude des caractères de production et de composition du lait de chèvre, sans prendre en considération des variables temporelles et alimentaires qui ont une incidence importante sur la sécrétion lipidique de la mamelle de chèvre comme l'ont montré DELAGE et al. (I967), FFHR et al. (I972) et SAUVANT et al. (I97I).

A partir des résultats des trente variables considérées, les analyses factorielles ont permis de dégager les quatre principaux facteurs de variation suivants : production, longueur de la chaîne carbonée des acides gras, opposition entre les couples d'acides $14: 0,16: 0$ et $\mathrm{I} 8: 0, \mathrm{I} 8: \mathrm{I}$ et opposition entre les caractères azotés et lipidiques du lait. Les autres facteurs interviennent surtout sur les variables dont la part de l'inertie expliquée par ces quatre premiers facteurs de variation est relati- 
vement faible. Ces autres facteurs pourraient être l'imprécision des techniques de mesure dans le cas des acides $\mathrm{I} 8: 3$ et $\mathrm{I} 9:$ o et la variabilité individuelle pour les caractères à forte héritabilité que sont les teneurs en matières azotées et en matières grasses du lait.

La matrice des corrélations montre une opposition entre la production de lait et les caractères de concentration en matières grasses ou azotées. Une telle relation entre la production laitière et le taux butyreux dont JARRIGE et JOURNE'T (I958) ont discuté la signification a été souvent observée au niveau interindividuel et intraindividuel sur les troupeaux de vaches. Chez la Chèvre, elle a été aussi constatée à partir des résultats du contrôle laitier (DISSET et al., I966). JARRIGE et RosSETTTI (I957) par ailleurs, ont enregistré une corrélation le plus souvent négative au cours de la lactation entre la production laitière et la teneur en matières azotées. Cette opposition entre les caractères de production et de concentration est en fait la résultante de phénomènes que les analyses factorielles ont permis de décomposer. Sur le premier axe, l'analyse des correspondances (graph. 4) oppose nettement les concentrations et la production, alors qu'en composantes principales (graph. I) le taux de matières azotées reste seul bien opposé à la production, tandis que le taux butyreux tend à être orthogonalà ce facteur. Par contre, sur le $2^{\mathrm{e}}$ axe en analyse des correspondances et $1 \mathrm{e} 4^{\mathrm{e}}$ axe en composantes principales qui sont indépendants de l'antagonisme production-concentration, les caractères azotés et lipidiques sont nettement opposés et la production du lait est très proche du caractère azoté. Il est possible que des facteurs alimentaires expliquent cette configuration des caractères déià observée par LEFEBVRE et al. (I97I). En effet, la consommation de foin non haché et de granulés à base de luzerne et de céréales, et de ce fait, le rapport $\frac{\text { Fourrages longs }}{\text { Aliments granulés }}$ pouvaient varier. Or, quand ce rapport diminue, le taux butyreux du lait de chèvre baisse alors que la teneur en matières azotées a tendance à s'élever ( $\left.\mathrm{FEHR}_{\mathrm{E}} \mathrm{I} 97 \mathrm{I}\right)$. De plus, la production laitière peut augmenter avec la teneur en matières azotées, notamment si une part des fourrages de la ration est distribuée sous forme granulée ; ce qui permet en général d'élever le niveau de consommation (JoURNET et JARRIGe, I967). Le fait que le taux butyreux moyen des 55 chèvres est inférieur de 7 points à la moyenne nationale et que la teneur en matières azotées est égale à cette moyenne nationale tendrait à indiquer que certains animaux ont utilisé la possibilité qui s'offrait à eux de réduire leur consommation de fourrages longs en ingérant de grandes quantités d'aliments granulés.

Dans ces conditions, il est fort possible que les techniques utilisées ont discriminé les variations des paramètres dues à des facteurs physiologiques individuels et à des facteurs alimentaires.

Dans les graphiques I et 4 , les acides gras à I 8 atomes de carbones semblent plus. liés à la production laitière que les autres acides. Il est vraisemblable que les chèvres fortes productrices consommaient plus d'aliments granulés, donc à la fois plus d'énergie et d'acides gras en $\mathrm{C}_{18}$. Dans ces conditions, il n'est pas étonnant que la production. laitière et les pourcentages d'acides en $\mathrm{C}_{18}$ aient des comportements voisins.

Par ailleurs, la teneur en matières azotées se situe, sur les graphiques I, 4, au voisinage des acides myristique et palmitique qui sont les plus sensibles à l'apport d'acide acétique à la mamelle. LINZELI (I968) indique que certains acides aminés des protéines du lait sont synthétisés dans la mamelle à partir d'acide acétique et 
d'autres à partir de glucose. Ainsi, le comportement voisin de la teneur en matières azotées et des acides en $\mathrm{C}_{14}$ et $\mathrm{C}_{\mathbf{1 6}}$ sur certaines composantes pourrait s'expliquer éventuellement par l'effet d'un précurseur commun. L'acide acétique ajouté à une ration riche en aliments granulés et pauvre en fourrages longs augmente en effet la concentration en matières azotées et en acides myristique et palmitique du lait de chèvre (FEHR et al., I972).

Les acides gras de même origine métabolique tels que les acides de 8 à I 2 atomes de carbone et les acides en $\mathrm{C}_{18}$ ont, quelle que soit la métrique, une représentation groupée. En revanche, les acides dont l'origine est plus complexe comme 1'acide palmitique et dans une moindre mesure, l'acide myristique, ont des comportements plus particuliers.

Dans les plans (I-2) et (2-3) en composantes principales (graph. I et 2) et sur l'axe 4 des correspondances (graph. 5), le pourcentage d'acide palmitique est opposé à ceux des acides en $\mathrm{C}_{18}$, celui de l'acide myristique se plaçant dans une position intermédiaire entre les acides plus courts et l'acide en $\mathrm{C}_{16}$. Cette opposition entre les acides en $\mathrm{C}_{14}$ et $\mathrm{C}_{16}$ et en $\mathrm{C}_{18}$ semble être le reflet de l'inhibition de la synthèse des acides gras à partir de précurseurs à 2 ou 4 atomes de carbone lorsque la mamelle dispose d'acides d'origine alimentaire, donc essentiellement en $\mathrm{C}_{18}$, en grande quantité (HIBITT et al., I966; MoORE et STEELE, I968; FEHR et al., I972). Les graphiques I et 2 (droites $D_{1}$ et $D_{2}$ ) confirment que cette inhibition se répercute surtout sur le pourcentage de l'acide palmitique et pour une moindre part, sur celui de l'acide myristique (FEHR et al., I972; SAUVANT et al., I97I).

En revanche, par rapport aux axes 2 et 4 en composantes principales (axes 2 et 3 des correspondances) l'acide palmitique a un comportement proche de ceux des acides stéarique et oléique. Or, une partie de 1'acide palmitique du lait comme 1'ensemble des acides en $\mathrm{C}_{18}$, n'est pas synthétisée par la mamelle (ANNison et al., I967) mais provient des lipides plasmatiques. Ce fait apparaît une raison suffisante pour que les acides en $\mathrm{C}_{18}$ et $\mathrm{C}_{18}$ aient des comportements comparables par rapport à certains axes. Ainsi, la méthodologie employée aurait permis en dehors de toute technique d'investigation physiologique de discriminer les deux origines métaboliques différentes de l'acide palmitique du lait.

Cette double origine n'existe pas pour l'acide myristique et se traduit donc par une opposition systématique entre les proportions de $C_{18}$ et $C_{14}$ dans les graphiques I, 2 et 3 .

Il est aussi intéressant de noter que dans les graphiques 3 et 4 , 1'acide stéarique paraît beaucoup plus en étroite relation avec les caractères lipidiques, alors que la position des acides en $\mathrm{C}_{18}$ insaturés s'en écarte sensiblement. Or, il a été mis en évidence qu'une graisse animale ou l'acide stéarique ajouté au régime est plus favorable qu'une huile végétale ou un acide insaturé à la sécrétion de matières grasses par la mamelle (Rook et STORRY, I964; MOORE et STEELE, I968).

Par ailleurs, la position des proportions des acides linoléique et linolénique sur le $4^{\mathrm{e}}$ axe en composantes principales ou sur le $2^{\mathrm{e}}$ axe de $1^{\prime}$ analyse des correspondances confirme l'hypothèse de causalité alimentaire qualitative qui a déjà été avancée. En effet, lorsque les vaches consomment un régime riche en aliments concentrés ou renfermant des fourrages sous forme concentrée, les fermentations du rumen sont modifiées, la production laitière et la teneur en matières azotées ont plutôt tendance à augmenter alors que le taux butyreux baisse très nettement ; de plus, les pour- 
centages en acides gras insaturés dans le lait s'élèvent, en revanche, ceux des acides gras saturés, notamment en $\mathrm{C}_{10}$ et $\mathrm{C}_{18}$ diminuent (KING et HEMKEN, I962, BEITZ et DAvis, I964. JORGENSEN et al., I965 ; STORRY et RoOK, I965 ; OpsTVEDT et RoNNING, I967; GHADAKI, I968). Dans ces conditions, il n'est pas surprenant que les pourcentages des acides linoléique et linolénique soient les plus proches des caractères de production laitière (fig. I et 4 ) et que celui de l'acide stéarique soit le plus voisin du taux butyreux (fig. 3 et 4 ).

Comme nous l'avons remarqué précédemment, trois groupes d'acides peuvent être distingués suivant leur comportement dans les plans de représentation : de $C_{8}$ à $\mathrm{C}_{12}, \mathrm{C}_{16}$ avec le plus souvent $\mathrm{C}_{14}$ et les $\mathrm{C}_{18}$. A la suite de divers travaux nutritionnels, Garton et Duncan (I956), GERson et al. (I966), Fisher et al. (I967), ont aussi été conduits à faire cette distinction à la différence qu'ils placent le plus souvent les acides de $\mathrm{C}_{12}$ à $\mathrm{C}_{16}$ ensemble. Les méthodes d'analyses factorielles utilisées semblent proposer une méthode de classification différente des acides selon leur affinité. En effet, si cette distinction en trois groupes d'acides peut être bien mise en évidence, chaque acide a en fait, un comportement propre en relation avec la longueur de sa chaîne, de son degré de saturation ou d'autres facteurs métaboliques encore inconnus.

Bien qu'étant le caractère le moins bien expliqué par les composantes principales (tab1. 5), le numéro de lactation reste le plus souvent proche de la production laitière. Cette observation peut s'expliquer par l'âge des chèvres qui ne dépasse pas 4 ans. En effet, DisSET et al. (I966 et I970) ont constaté que la production laitière ne progresse que jusqu'à la quatrième lactation chez la Chèvre.

Dans cette étude, les deux méthodes d'analyses ont permis d'isoler les mêmes facteurs principaux de variation des résultats mais dans des ordres différents d'importance. Cependant, comme HENRY et al. (I97I) l'ont observé, l'interprétation détaillée des résultats n'a pas permis de déceler l'opposition entre les conclusions suggérées par les deux techniques. Toutefois, nous avons constaté que l'analyse des correspondances travaillant sur les profils, a une fonction de discrimination beaucoup plus nette, notamment entre les caractères de production et de concentration et aussi entre les caractères azoté et lipidique du lait. Cette faculté jointe à l'absence d'hypothèse sur les lois des variables permet d'utiliser efficacement des codages a priori sur des caractères qualitatifs. Par contre, l'analyse en composantes principales normées par son choix de la métrique euclidienne classique, permet d'interpréter et de quantifier les résultats suivant les concepts biométriques familiers (corrélation, variance, orthogonalité...) tout en lui laissant la possibilité de faire un codage a posteriori sur les observations. Enfin la projection simultanée des observations et des variables sur les mêmes plans factoriels, peu admise en composantes principales, facilite grandement l'interprétation des sorties des correspondances.

Ce type d'analyse déjà utilisé en génétique pour des caractères zootechniques classiques (LEFEBVRE et al., I97I, OLLIVIER, I970) peut permettre d'affiner le choix des caractères de base. En effet, il semble par exemple dans notre cas, possible malgré "l'effet régime " de sélectionner des animaux produisant un lait riche en un groupe d'acides gras donnés (graph. 4) ou bien de prévoir vers quel type de composition en acides gras du lait conduira une sélection sur la production laitière, le taux butyreux ou la teneur en matières azotées et donc de discerner les conséquences technologiques ou diététiques de telles orientations.

Ces méthodes paraissent particulièrement aptes à déceler les composantes d'un 
fait métabolique. Ainsi, dans le cas présent, les deux origines de l'acide palmitique ont pu être discernées par simple observation des résultats d'un troupeau conduit dans des conditions normales. Elles ont, par ailleurs, l'intérêt de pouvoir classer les facteurs par ordre décroissant d'importance. Certes, ces techniques d'analyse ne sont pas habilitées à donner une explication métabolique définitive, mais elles semblent être d'excellentes génératrices d'hypothèses appelées à être vérifiées par la suite.

Ces techniques biométriques, alliées au progrès du traitement de 1'information nous ont donc permis d'avoir une vue synthétique réelle des multiples relations dans un ensemble de caractères. De ce fait, l'apparition de ces méthodes d'analyse devrait modifier en recherche métabolique, nutritionnelle ou zootechnique tout au moins, le comportement méthodologique tendant à limiter le nombre des caractères étudiés simultanément pour éviter les difficultés d'interprétation généralement insurmontables.

Reçu pour publication en juillet 1972.

\section{REMERCIEMENTS}

Nous remercions M. Ricondeau d'avoir bien voulu accepter de mettre à notre disposition, le troupeau caprin du Domaine de Galle (1 8 - Avord) pour cette étude.

\section{SUMMARY}

\section{INTERRELATION BETWEEN CHARACTERS OF YIELD AND GOAT MILK LIPID COMPOSITION STUDIED WITH TWO METHODS OF FACTOR ANALYSIS}

The relation between milk yield, butterfat percentage and yield, protein content and yield, percentage and yield of milk fatty acids were studied with two methods of multivariate analysis on goats which had kidded about three months previously. The two methods of analyses used were principal component and correspondance analysis. They are described in order to show their respective originalities.

From the thirty variables considered, factor analysis reveal four main variation factors: yield, length of fatty acid carbon chain, opposition of $\mathrm{r} 4: 0$ and $\mathrm{I} 6: \mathrm{o}$ acids and $\mathrm{I} 8: 0$ and $\mathrm{I} 8: \mathrm{I}$ acids, and opposition of milk protein and lipid characteristics.

We were able to "visualize " very clearly in our experimental conditions the relation between yield and composition characters and between different fatty acids, milk yield, and fat percentage or protein content. Some of these relations may be explained because of our present knowledge of metabolism.

Three types of milk lipid composition have been determined within the goat population studied : milks rich in $8: 0$, Io: 0 , I2:o acids, in palmitic acid and in $\mathrm{C}_{18}$ acids.

These factor analysis methods could be used in the selection of goats of a given type of milk composition when the effects of that selection on other characters is known.

Although the detailed interpretation of results does not show an opposition between the conclusions obtained by the two methods, the advantages of each of them and their capacity to meet the requirements of metabolic and animal science research are discussed.

\section{RÉFÉRENCES BIBLIOGRAPHIQUES}

Annison E. F., Linzell J. L, Fazakerley S., Nichols B. W., 1967. The oxidation and utilization of palmitate, stearate, oleate and acetate by the mammary gland of the fed goat in relation to their overall metabolism and the role of plasma phospholipids and neutral lipids in milk fat synthesis. Biochem. J., 102, 637-647. 
Benzecri J. P., I964. Cours de linguistique mathêmatique. Faculté des Sciences de Rennes.

CoRteel J. M., I971 (a). L'insémination artificielle caprine. Bull. Techn. Inf., 257, x53-156.

Corteel J. M., I97i (b). La maîtrise du cycle sexuel chez le chevrette et chez la chèvre. Bull. Tech. Inf., 257, I75-I80.

Delage J., Fehr P. M., I967. Influence des lipides alimentaires sur la sécrétion des acides gras par la mamelle de chèvre. Ann. Biol, anim. Biochim. Biophys., 7, 437-444 et 445-457.

Disset R., I97I. Communication personnelle.

Disset R., Ricordeau G., Cattin-Vidal P., i 966 . Performances laitières de chèvres contrôlées en I964. La chèvre, 9, 4I, 8-I2.

Disset R., Sigwald J.-P., Ricondeau G., I97o. Analyse des résultats du contrôle laitier caprin en I968. Patre, $\mathrm{n}^{\circ}$ I76, 8-I5.

FEHR P.-M., I97I. Utilisation des fourrages granulés par la chèvre laitière. Ann. Zootech., 20, 305-320.

Fenr P.-M., Sauvant D., Delage J., I972. Effets isolés et combinés des acides acétique, propionique, laurique et stéarique sur la sécrétion lipidique de la mamelle de chèvre. Ann. Biol. anim. Biochim. Biophys., 12, 289-306.

Fisher L. J., Elliot J. M., Corse D. A., I967. Fatty acid composition of bovine milk fat as influenced by intravenous infusion of propionate or glucose. J. Dairy Sci., 50, 53-56.

Folley S. J., Mav NaUght M. L., I96r. Biosynthesis of milk fat in milk. The mammary gland and its secretion. Vol. 1, 441-482, éd. by Kon S. K. and Cowie A. T. Academic Press inc. New York.

GHADAKI M. B., I968. Infuence de quelques facteurs alimentaires sur la sécrétion des principaux acides gras du lait de vache. Thèse Fac. Sci. Clermont-Ferrand (France).

Garton G. A., Duncan R. H., 1956. The fatty acid composition of milk fats from beef cows fed on different winter rations. J. Sci. Fd. A gric., 7, 735-739.

Garton G. A., I963. The composition and biosynthesis of milk lipids. J. Lipid Res., 4, $237-254$.

Gerson T., Wilson G. F., Singh H., Shorland F. B., ig66. Origin of the glyceride fatty acids of milk fat. J. Dairy Sci., 49,680-68r.

Henry Y., Rérat A., Tomassone R., I97I. Étude du besoin en lysine du porc en croissance-finition. Application de l'analyse multidimensionnelle. Ann. Zootech., 20, 52 I-550.

Hibits K. G., I966. Some factors involved in the control of fatty acid synthesis in the lactating bovine mammary gland. Biochim. Biophys. Acta, 116, 56-66.

Hotelling H., I933. Analysis of a complex of statistical variables into principal components. J. Educ. Psych., 24, 4I7-44I.

Jarrige R., Rossetri C., I957. Études sur les variations de la richesse en constituants azotés des laits de vache. II. Evolution au cours de la lactation et de la gestation. Ann. Zootech., 6, 4I-63.

JARRIGE R., I958. Influence des facteurs alimentaires et climatiques sur la teneur en matières grasses du Iait. Journées Scientifiques des Corps Gras Alimentaires. $2^{\mathbf{e}}$ série : 9-12 décembre 1958 . Publication C. N. R. S. I3, Quai Anatole-France, Paris $7^{\mathrm{e}}$, p. A 233-277.

Jones E. A., 1969. Reviews of the progress of dairy science. Section A. Recent developments in the biochemistry of the mammary gland. J. Dairy Res., 36, I45-I66.

Jorgensen N. A., Schultz L. H., BARR G. R., I965. Factors influencing milk fat depression on rations high in concentrates. J. Dairy Sci., 48, I03 I-39.

JoURnet M., JARRIGE R., I967. Utilisation đes aliments broyés et agglomérés par les bovins. II. Utilisation comparée par la vache laitière du foin de luzerne condensé et du foin de luzerne normal associés à de l'ensilage et des betteraves. Ann. Zootech., 16, 27I-289.

King R.-L., Hemken R.-W., 1962. Composition of Milk Fat Produced on Pelleted Hay and heated Corn. J. Dairy Sci., 45, 1336-42.

Lebart L., FÉnelon J. P., I97I. Statistiques et informatique appliquées. Dunod (Paris).

Lefebvre J., Ricordeau G., Lebeaux M.-O., i97I. Correspondance entre éléments de conformation et de production laitière chez les bovins Montbéliards. $X^{\mathrm{e}}$ Congrès international de Zootechnie, Versailles $20-23$ juillet I97I. Thème VII.

Lefort G., I968. Cours de statistiques à l'Institut National Agronomique.

Linzelt J. L., I968. The magnitude and mechanism of the uptake of milk precursors by the mammary gland. Proc. Nutr. Soc., 27, 44-52.

Moore J.-H., Steele W., I968. Dietary fat and milk secretion in the cow. Proc. Nutr. Soc., 27, 66-70.

Ollivier L., I97o. L'épreuve de la descendance chez le porc Large White français de I953 à I966. I. Analyse de la variation. Ann. Génét. Sél. anim., 2, 3II-324.

Opstvedt J., Ronning M., I967. Effect upon Lipid Metabolism of Feeding Alfalfa Hay or Concentrate ad Libitum as the Sole Feed for Milking Cows. J. Dairy Sci., 50, 345-54.

Rook J.-A.-F., SToRry J. E., I964. Energy nutrition and milk secretion in the dairy cows. Chem. Ind., 43, х778-I787.

Sauvant D., Fehr P.-M., Delage J., I97i, Études des interactions des effets des acides acétique et stéarique sur le rendement lipidique de la mamelle de la chèvre laitière. $X^{\mathbf{e}}$ Congrès international de Zootechnie, Versailles 20-24 juillet I97I. Thème VII. 
STORRY J. E., Rook J. A. F., I965. The effects of a diet low in hay and high in flaked maize on milkfat secretion and on the concentrations of certain constituents in the blood plasma of the cow. Brit. J. Nutr., 19, IoI-109.

Storry J. E., Rook J. A. F., Hali A. J., 1967. The effect of the amount and type of dietary fat on milk fat secretion in the cow. Br. J. Nutr., 21, 425-438.

Stull J. W., Brown W. H., Mohammed K., I966. Changes in fatty acids of milk fat caused by intravenous administration of acetic, propionic and butyric acids. J. Dairy Sci., 49, 737.

Tomassone R., I97o. L'analyse factorielle des correspondances. III $I^{\mathrm{e}}$ Congrès $J . U . F . R . O$. du Groupe des Statisticiens forestiers, 7-I I sept. I970, I. N. R. A., Jouy-en-Josas. 